and their experiences with transport risks. Additional information about teaching practices and implementation issues were explored in interviews with 13 teachers. A psychological theory was selected to operationalise the design of the programmes that drew on such preparatory data. The programme, Skills for Preventing Injury in Youth was evaluated with 197 participating and 137 control students (13-14 year olds). Results showed a significant difference between the intervention and control groups from baseline to 6-month follow-up in a number of transport-related risk behaviours and transport-related injuries. The programme thus demonstrated potential in reduce early adolescents transport risk behaviours and associated harm. Discussion will involve the implications of the development research process in designing road safety interventions.

\title{
0329 CREATING AN EFFECTIVE PREVENTION CURRICULUM TO REDUCE EARLY ADOLESCENT TRANSPORT RISKS AND INJURY
}

L Buckley*, R Chapman, M Sheehan Correspondence: Queensland University of Technology, 130 Victoria Park Road, Kelvin Grove, Queensland 4059, Australia

\subsection{6/ip.2010.029215.329}

Adolescent injury is a significant health concern and can be a result of the adolescents engagement in transport-related behaviours. There is however significant planning and formative research needed to inform prevention programme design. This presentation reports on the development and evaluation of a curriculum programme that was shown to be effective in reducing transport-related risks and injuries. Early adolescents report injuries resulting from a number of transport-related behaviours including those associated with riding a bicycle, a motorcycle, and as a passenger (survey of 209 Year 9 students). In focus groups, students $(n=30)$ were able to describe the context of transport risks and injuries. Such information provided evidence of the need for an intervention and ecologically valid data on which to base programme design including insights into the language, culture and development of adolescents 